\title{
ULTRASONOGRAPHY IN \\ PHYSIOTHERAPY AND \\ REHABILITATION
}

Barassi Giovanni ${ }^{1}$, Guerri Sergio ${ }^{2}$, Tavani Roberta ${ }^{3}$, Ricucci Giampiero ${ }^{4}$, De Luca Giorgia ${ }^{5}$, Rabini Alessia ${ }^{6}$, Colombo Antonio ${ }^{6}$, Di Iorio Angelo

\author{
${ }^{I} P h D$, Coordinator of the Physiotherapy Center for Rehabilitation and Reeducation (Ce.Fi.R.R.) venue "G. d'Annunzio" \\ University of Chieti-Pescara (Italy). \\ ${ }^{2} M D$, Sonography Specialist, Sector of Clinical Evaluation and Diagnosis of Ce.Fi.R.R. venue "G. d'Annunzio" \\ University of Chieti-Pescara (Italy). \\ ${ }^{3} \mathrm{MD}$, Gastroenterolgy and Sonography Specialist, Ultrasound Diagnostic Center Chieti (Italy). \\ ${ }^{4}$ BSc, Health Manager of the Rehabilitation and Sonography-guided Physiotherapy Center "Fisiofilippone" (Italy); \\ Technical Consultant for the Chieti Court (Italy). \\ ${ }^{5}$ BSc, Rehabilitation and Sonography-guided Physiotherapy Center "Fisiofilippone" (Italy). \\ ${ }^{6}$ Department of Cardiovascular Diseases, Gemelli Molise-Cmapobasso (Italy). \\ ${ }^{7}$ Associate Professor Department of Aging Sciences, “G.d'Annunzio” University Chieti-Pescara.
}

KEYWORDS: Ultrasonography, Echography, Ultrasonographic Imagings, Physiotherapy,

\section{ABSTRACT}

There is an interrelation with ultrasound / physiotherapist and the duty of the physiotherapist to know how to perform ultrasound examinations alone, not for diagnostic purposes, to follow the evolution of the therapeutic cycle of physiotherapy.

For this reason, ultrasound image analysis (US) is a promising non-invasive approach that uses load-dependent changes in the intensity of the echo to characterize the rigidity of muscle and tendon tissue.

The purpose of this contribution is to improve the use of ultrasound images (US) and the role of the physiotherapist, who are able to detect localized changes, in particular in stiffness of the tendon due to partial and full-thickness tendon tears. Image intensity information is less sensitive for identifying load transmission variations resulting from partial thickness cuts initiated on the joint side.

Ultrasound images can be useful for quantitatively assessing the variations dependent on the tendon load and muscle stiffness in physiotherapy and that the interruption of the behavior of the acousto-elastic ultrasound images can be indicative of substantial damage to the muscle or tendon.

\section{INTRODUCTION}

Rehabilitative UltraSound Imaging (RUSI), ultrasound for rehabilitation purposes, is a procedure used by physiotherapists to evaluate the morphology and functionality of muscle and related soft tissues during exercise and physical activities. It is used to assist the application of therapeutic interventions, providing important feedback to the patient and physiotherapist (Teyhen, 2006; Day \& Uhl, 2013).

One tool that has the potential to help improve physiotherapists' ability to evaluate and treat motor control disorders is the use of rehabilitation ultrasound imaging (RUSI) based on its ability to provide real-time visual feedback of morphology. underlying muscle and function for both the patient and the physiotherapist. Although research and clinical applications of this emerging technology have steadily grown, ultrasound, a diagnostic method that has become increasingly popular in the past 50 years, has nowadays reached high standards of technique and image quality.

The ultrasound image is produced using an ultrasound beam that is generated by a probe through the physical principle of piezoelectricity. They penetrate the tissues under examination, are to varying degrees deflected or reflected and subsequently picked up by the emitting probe (this time with the receiving function) which, through the system processor, generates the image that can be displayed on the screen or printed.
The ultrasound examination is therefore a method of investigation free of ionizing emissions, therefore free from the risk of side effects on biological systems.

Ultrasound applications cover all human tissues, ultrasounds of the abdomen, soft tissues, breast, thyroid gland and musculoskeletal-skeletal system can therefore be performed.

In the recognition of pathologies of ultrasound evaluation relevance, the concept of echogenicity, that is the ability of soft tissues to reflect ultrasound or not, is fundamental. A greater echogenicity, typical of thickened tissues (eg fibrosis) will therefore give rise to very clear images of the structures explored (hyperechoic); vice versa in the tissues where edema prevails or in blood or serous collections, the image will tend to be darker than the average (hypoechoic). In applications of the skeletal system, ultrasound examination finds its greatest impediment in the impossibility of being able to explore the structure of the bone, since ultrasound does not have enough energy to go beyond the cortical layer; the bone will therefore be represented exclusively as a profile (profile that in any case can provide important information on its integrity), without details of the underlying tissue.

On the contrary the ultrasound excels in the visualization of the muscle-ligament systems; it is therefore fundamental in the diagnosis of lesions, inflammations, the presence of blood or serous 
collections and, if carried out by an expert operator, also any meniscal lesions.

Limitation of the ultrasound investigation is the strict interdependence with the operator's abilities, the ultrasound examination is therefore highly dependent operator; inexperience, approximation in carrying out the examination, an insufficient knowledge of the anatomy, can lead to a failure or an incomplete diagnosis (Hebert et al., 2009).

Ultrasound for rehabilitation purposes is a tool that the physiotherapist uses for functional assessment procedures (L. 251/00) to carry out the skills of the profession and does not involve any clinical risk for the user. The ultrasound instrument is therefore not used to make a diagnosis of pathology, but to make the assessments of the morphology, size and functionality of muscle structures and soft tissues more precise and reliable, which are commonly performed by the professional through patient inspection and manual measurement. A detailed and reliable assessment allows you to set more individualized treatment plans and provide more patient feedback while performing the exercises.

Despite the synthetic technical notions mentioned above, it is evident that ultrasound investigation is valuable in physiotherapy; in fact, it allows the diagnosis of almost all muscle-ligament pathologies. It is very efficient in identifying muscle and ligament inflammation, complete, incomplete, or fibrillary lesions, blood and / or serous collections, meniscal lesions and any changes in the bone profile.

For the physiotherapist, therefore, a well-done ultrasound diagnosis proves to be a valuable tool for recognizing the pathology that it will have to treat and indispensable in planning an adequate therapeutic plan.

At the end of the therapy cycle, a new ultrasound examination, better if conducted by the operator who made the diagnosis for better diagnostic continuity, will confirm the recovery or highlight any other problems that have emerged in the meantime.

SCIENTIFIC LITERATURE REVIEW

Various experiences have been collected in the literature that enhance the idea of the use of musculoskeletal ultrasound by the physiotherapist.

We feel we can deny the assumption that the physiotherapist should be a passive user of the ultrasound investigation. If it is true that the degree in physiotherapy does not recognize the legal capacity to make a diagnosis, the physiotherapist therefore cannot comment on the medical recognition of a pathology of the musculoskeletal-skeletal system; equally true is that it would be desirable an ever-greater ability of the physiotherapist to know how to carry out ultrasound examinations for evaluation and exclusive driving purposes during the course of the therapy cycle.

Already in 2006, at a congress held in San Antonio (USA), the RUSI (Rehabilitative UltraSound Imaging) method was defined internationally. The RUSI method defines that the physiotherapist can use ultrasound to follow the effectiveness of the chosen therapeutic interventions.

Primary factor in the execution of the ultrasound examination and in the development of physiotherapy, obviously an absolute and essential knowledge of the anatomical structures of the organism remains. Having said that, knowledge of the physical and practical principles of the operation of the ultrasound machine remains extremely indispensable; in fact, the use of a specific probe or the use of a specific examination frequency depends, always and in any case, beyond the theoretical knowledge, on the practical experience of the examiner.

Having clarified the boundaries within which the ultrasound activity can be carried out by the individual physiotherapist, it remains to be specified which can be its practical application.

It consists in the possibility of following the evolution of the patient's condition, even with each single application (Warden \& McMeeken, 2002).

Thus, a blood or serous collection can be assessed during the course of its resorption, a muscle injury in its healing process and so on. It goes without saying that any therapy errors can thus be corrected promptly without waiting for the end of the therapy cycle and the check by the sonographer.

In this regard, the diagnosis of healing by the sonographer is fundamental, to protect the patient and the physiotherapist (Jopowic et al., 2017).

Recently, ultrasound has been used to evaluate tissue tension and other mechanical properties (Samani et al., 2007).

Many researchers have attempted to evaluate tissue tension and mechanical properties using elastography, a technique originally proposed by Ophir et al. (Ophir et al., 1991) which maps deformation distributions in problems resulting from surface compression; since deformation is inversely related to stiffness, elastography is an indirect method of estimating tissue stiffness (Doyley et al., 2000).

Although researchers have used this technique to identify tissue properties, current elastographic methods have some inherent limitations.

The elastography traces the uneven reflections of the echo ("speckles" resulting from heterogeneity of the tissues) in the ultrasound images during loading (usually using the transducer to apply compression).

The deformation information calculated using the distortions of these reflectors is related to the mechanical properties by post hoc mechanical analysis. A limitation of the elastography is that it is intrinsically linear, if the material properties and the speed of the ultrasonic wave do not change during the measurement of the deformation, which limits the analyzes to small increases in compression.

When soft tissues were tested with major and therefore nonlinear deformations in stiffness, significant errors occurred (Zhi et al., 2007).

This is problematic in soft tissues as in tendons as it is not linear in stiffness and subjected to relatively large deformations during the activity, reaching efforts of several percent (elastography works best when the increases in effort are limited to less than $1 \%$ ).

Another limitation is the commonly used method of compression testing; the tendon is loaded in tension in vivo; therefore interesting mechanical information is lost if only the transverse compressive loads are considered.

These restrictions associated with standard elastographic methods limit its applicability to the tendon. Finally, elastography measures tension. Additional data, both stress and stiffness, are needed to fully describe mechanical behavior (Zhi et al., 2007). 
The theory of acoustoelasticity, developed by Hughes and Kelly (Hughes \& Kelly, 1953), is based on the principle that the acoustic properties of a material are altered when the material is deformed and loaded, just as a change in tension alters the height of a string. guitar.

The changes in acoustic properties caused by elastic deformation can be measured as a change in the propagation speed of the wave or in the amplitude of the reflected wave (Kobayashi \& Vanderby, 2005; Kobayashi \& Vanderby, 2007).

Kobayashi and Vanderby derived the acoustoelastic relationship between reflected wave amplitude and mechanical behavior (stiffness and stress dependent on deformation) in a deformed material, almost incompressible using the A-mode 1-D ultrasound. Despite signal processing, this phenomenon also occurs in B-mode 2-D ultrasound, as the tension of the tendon increases the intensity of the reflected ultrasonic echoes, leading to a brighter ultrasound image in B-mode. Examples of this acoustoelastic effect in soft tissues have been reported in the literature (Potter et al., 2012).

Even the prices for the acquisition of the ultrasound machine must not be frightening as as a minimum equipment for the physiotherapist you can safely think of a good device to equip your own studio.
Fundamental in the research of the device are the good general conditions but above all the provision of at least two probes that are intact and do not create images with black spaces (the linear probe for highdefinition surface structures and convex, probe with less definition of image but with greater viewing depth).

The need for a photo printer is not considered essential unless the operator intends to keep track of his work or wants to dedicate himself to publication.

\section{CONCLUSIONS}

Evaluative ultrasound is absolutely within the physiotherapist's reach, it is a precious and irreplaceable method of visualization in following the evolution of a cycle of physical therapy, providing objective and not only subjective feedback to it. Despite growing scientific evidence on the use of this practice, several questions remain. Future research needs to define in more detail the potential and limitations of this tool in measuring muscle function, and the factors that can influence muscle diameter as seen on ultrasound. Further studies are also needed to determine the minimum training standards required of physiotherapists to use and interpret the technique effectively.

\section{REFERENCES}

1. Day, J. M., \& Uhl, T. (2013). Thickness of the lower trapezius and serratus anterior using ultrasound imaging during a repeated arm lifting task. Manual therapy, 18(6), 588-593.

2. Doyley, M. M., Meaney, P. M., \& Bamber, J. C. (2000). Evaluation of an iterative reconstruction method for quantitative elastography. Physics in Medicine \& Biology, 45(6), 1521.

3. Hebert, J. J., Koppenhaver, S. L., Parent, E. C., \& Fritz, J. M. (2009). A systematic review of the reliability of rehabilitative ultrasound imaging for the quantitative assessment of the abdominal and lumbar trunk muscles. Spine, 34(23), E848-E856.

4. Hughes, D. S., \& Kelly, J. L. (1953). Second-order elastic deformation of solids. Physical review, 92(5), 1145.

5. Jopowicz, R., Jopowicz, M., Deszczyński, J. M., \& Deszczyński, J. (2017). Current Uses of Ultrasound Imaging in Musculoskeletal Rehabilitation. Ortopedia, traumatologia, rehabilitacja, 19(6), 503-511.

6. Kobayashi, H., \& Vanderby, R. (2005). New strain energy function for acoustoelastic analysis of dilatational waves in nearly incompressible, hyper-elastic materials. J. Appl. Mech., 72(6), 843-851.

7. Kobayashi, H., \& Vanderby, R. (2007). Acoustoelastic analysis of reflected waves in nearly incompressible, hyper-elastic materials: forward and inverse problems. The Journal of the Acoustical Society of America, 121(2), 879-887.

8. Ophir, J., Cespedes, I., Ponnekanti, H., Yazdi, Y., \& Li, X. (1991). Elastography: a quantitative method for imaging the elasticity of biological tissues. Ultrasonic imaging, 13(2), 111-134.

9. Potter, C. L., Cairns, M. C., \& Stokes, M. (2012). Use of ultrasound imaging by physiotherapists: a pilot study to survey use, skills and training. Manual therapy, 17(1), 39-46.

10. Samani, A., Zubovits, J., \& Plewes, D. (2007). Elastic moduli of normal and pathological human breast tissues: an inversiontechnique-based investigation of 169 samples. Physics in medicine \& biology, 52(6), 1565.

11. Teyhen, D. (2006). Rehabilitative Ultrasound Imaging Symposium, May 8-10, 2006, San Antonio, Texas. Journal of Orthopaedic \& Sports Physical Therapy, 36(8), A-1.

12. Warden, S. J., \& McMeeken, J. M. (2002). Ultrasound usage and dosage in sports physiotherapy. Ultrasound in medicine \& biology, 28(8), 1075-1080.

13. Zhi, H., Ou, B., Luo, B. M., Feng, X., Wen, Y. L., \& Yang, H. Y. (2007). Comparison of ultrasound elastography, mammography, and sonography in the diagnosis of solid breast lesions. Journal of ultrasound in medicine, 26(6), 807-815. 